\title{
A procedure for multi-objective optimization of tire design parameters
}

\author{
Nikola Korunović , Miloš Madić, Miroslav Trajanović and Miroslav Radovanović
}

Faculty of Mechanical Engineering, University of Niš, A. Medvedeva 14, Niš, Serbia

\begin{tabular}{l}
\hline C H R O N I C L E \\
\hline Article history: \\
Received September 92014 \\
Received in Revised Format \\
October 232014 \\
Accepted November 252014 \\
Available online \\
November 262014 \\
\hline Keywords: \\
Tire design \\
Multi-objective optimization \\
Pareto \\
Strain energy density \\
Finite element method \\
\hline
\end{tabular}

\author{
A B S T R A C T
}

The identification of optimal tire design parameters for satisfying different requirements, i.e. tire performance characteristics, plays an essential role in tire design. In order to improve tire performance characteristics, formulation and solving of multi-objective optimization problem must be performed. This paper presents a multi-objective optimization procedure for determination of optimal tire design parameters for simultaneous minimization of strain energy density at two distinctive zones inside the tire. It consists of four main stages: pre-analysis, design of experiment, mathematical modeling and multi-objective optimization. Advantage of the proposed procedure is reflected in the fact that multi-objective optimization is based on the Pareto concept, which enables design engineers to obtain a complete set of optimization solutions and choose a suitable tire design. Furthermore, modeling of the relationships between tire design parameters and objective functions based on multiple regression analysis minimizes computational and modeling effort. The adequacy of the proposed tire design multi-objective optimization procedure has been validated by performing experimental trials based on finite element method.

(C) 2015 Growing Science Ltd. All rights reserved

\section{Introduction}

Tire is a complex structure designed for adverse exploitation conditions. A new tire design must satisfy numerous design requirements, which are dictated by rigid safety and environmental regulations as well as by constantly evolving performance demands and new trends. Therefore, tire design is a challenging task that mostly relays on designer's experience. Ever changing market demands, expensive physical prototypes and production uncertainties do not allow it to become a routine process. Significant progress in tire design has been achieved by introduction of virtual tire prototyping, which is mostly based on finite element method (FEM). Nevertheless, virtual prototyping is mostly performed by trial-and-error approach, which inevitably consumes a considerable portion of design process and still does not guarantee that the optimal results are obtained. From previous discussions, it may be concluded that tire design represents an excellent field for application of design optimization methods. This claim is supported lately by increased scientific interest in the area. Tire performance is evaluated through a large set of performance characteristics, such as dry/wet handling and traction, endurance, wear resistance, ride comfort, rolling resistance, aquaplaning, weight, etc. (Gent \& Walter, 2006). In a multi-objective tire optimization task a number of performance characteristics are simultaneously minimized or maximized within acceptable ranges. Objective functions are defined using global or local values of

* Corresponding author. Tel: +381 18500660, Fax: +38118500660

E-mail: nikola.korunovic@masfak.ni.ac.rs (N. Korunović)

(C) 2014 Growing Science Ltd. All rights reserved.

doi: $10.5267 /$ j.ijiec.2014.11.003 
stress, strain, energy, or some other physical quantities that directly influence the performance characteristics. Since tires carry out many functions and many of them have tradeoffs, it is important to find the combination of design variables that satisfy well-balanced performance in conceptual design stage (Koishi \& Shida, 2006). In order to effectively maximize tire maneuverability and durability, Cho et al. (2002) refined the conventional satisficing trade-off methods (STOM), which were originally proposed for the multi-objective structural optimization, by introducing a systematic aspiration-level adjustment procedure. Five design variables i.e. nodal radii at nodes situated on sidewall portion of carcass were considered in order to improve tire maneuverability and durability by optimization of carcass contour at the sidewall.

Koishi and Shida (2006) proposed a procedure to solve multi-objective tire design problems by integrating polynomial-based response surface models, multi-objective genetic algorithm (MOGA) and self-organizing map (SOM). By means of MOGA and SOM, a map of Pareto solutions, called the multiperformance map, was determined upon which one could easily find some combinations of tire design parameters that satisfy well-balanced performance. In the proposed multi-objective optimization procedure three geometrical tire design parameters, which define the shape of the tread, were considered. The goal was to improve uneven wear and wear life for both the front tire and the rear tire of a passenger car, which was formulated using four objective functions. Serafinska et al. (2013) proposed a multiobjective optimization procedure based on the aggregate objective function approach with consideration of fuzzy variables applied to structural tire design. Due to high computational effort, the numerical simulation model was subsequently substituted by an artificial neural network (ANN) based response surface approximation within the optimization loop. Belt angle, thickness of tread layer and number of cap plies were chosen as tire design parameters, while inner pressure, fiber spacing in carcass and stiffness of the tread compound were chosen as uncertain a-priori parameters. Within formulation of multi-objective optimization problem, two objective functions were considered. The first objective function was focused on achieving regular wear, which was obtained by providing a uniform contact pressure distribution in the tire-road contact zone. Within the second objective function, the occurrence of a fatigue crack was investigated by the evaluation of strain energy density.

The research presented in this paper was focused on determination of optimal tire design parameters to simultaneously minimize strain energy density at belt edge and chafer, which are known to influence tire durability. In this pilot study, axisymmetric FE model was used to simulate tire inflation process, in order to quickly test the methodology before a full study is performed using rolling analysis on a 3D FE model. In order to obtain the whole information in multi-objective solution and tire design parameters space, the proposed multi-objective optimization procedure was based on Pareto concept that enables design engineers to obtain a complete set of optimization solutions and chose a suitable tire design. In order to speed up the multi-objective optimization procedure and minimize computational and modeling effort, modeling of the relationships between tire design parameters and objective functions was based on multiple regression analysis (MRA). Finally, the adequacy of the proposed tire design multi-objective optimization procedure has been experimentally validated by performing FE experimental trials.

\section{Problem definition concerning tire design and FE modeling issues}

Typical structural components of a pneumatic tire are shown in Fig. 1. Structural components of the tire are either purely rubber or wire composites with rubber resin. In addition to complexity of tire structure, very stiff and very flexible materials are placed side by side in its interior. Abrupt stiffness changes occur in the tire, especially at belt edges, causing stress concentration. Belt edge is thus one of well known critical zones in tire construction. One of the other zones known to be critical is tire bead, where cyclic stress changes occur during tire rotation i.e. cyclic bead flexion. 


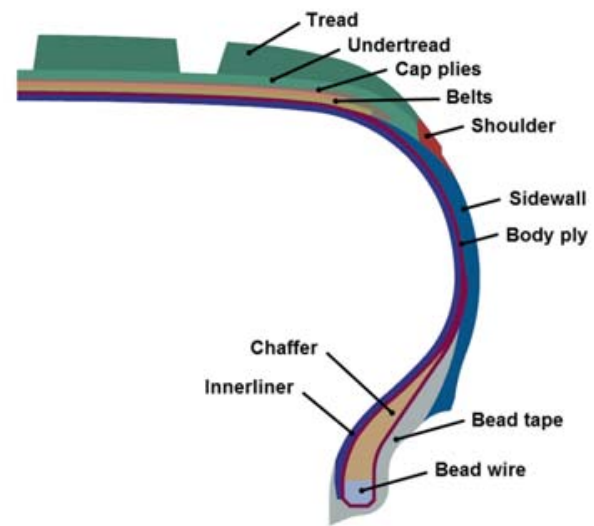

Fig. 1. Components of a radial tire

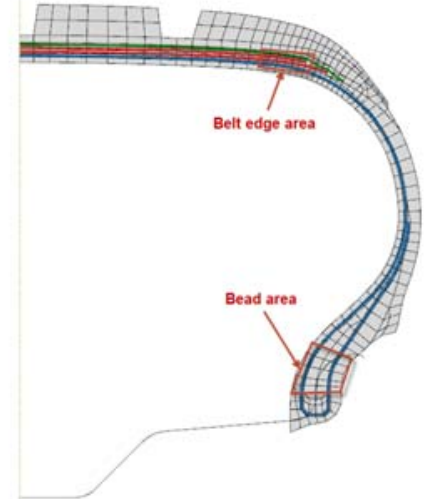

Fig. 2. Axisymmetric finite element tire model

As argued by De Eskinazi et al. (1990), strain energy density is seen to be a good indicator of complex stress-strain state at a given location inside the tire, taking into account material nonlinearities. Therefore, it should be a good predictor of rubber failure. It has already been used in tire optimization procedures by some authors, like Serafinska et al. (2013). For above reasons, the two objective functions considered in this paper are strain energy density at belt edge $\left(f_{1}\right)$ and strain energy density at chafer $\left(f_{2}\right)$. It is clear that in a realistic tire design optimization study, a rolling analysis of 3D FE tire model should be performed in order to get meaningful results related to behavior of the tire during its lifecycle. Nevertheless, as stated in the introduction, the main idea of the work presented in this paper was to perform a pilot study on a simple FE model that would allow the analyses to finish quickly. In this way, suitability of chosen optimization approach for tire design could be checked and compared with existing ones. Thus, axisymmetric model of one half of tire profile was selected (Fig. 2) and used for inflation analyses, in which three tire design parameters were changed: belt angle, belt cord spacing and elasticity of tread compound. From a number of parameters that are reported to have a significant influence on tire behavior (Olatunbosun \& Bolarinwa, 2004; Ghoreishy, 2006), i.e. on stress-strain state inside the tire, those design parameters were chosen as they were easiest to change inside the FE model.

Axisymmetric FE model used in the analyses contains an overall optimized mesh of one-half of tire profile, resulting from a convergence study. The chosen mesh yields only $0.65 \%$ less maximal stress in belt area than the finer one used in convergence study. The mesh contains an overall of 593 elements, 421 of which are linear hybrid axisymmetric elements with twist representing rubber components, 162 are embedded linear surface axisymmetric elements with twist containing rebar definitions to present tire reinforcements and 10 are linear axisymmetric elements representing bead wire. Rubber behavior is modeled using hyperelastic Yeoh material model, while cords are modeled as linear elastic material. Similar FE model is described in detail in (Korunović et al., 2007). Inflation pressure was 0.23MPa.

\section{Multi-objective optimization procedure}

The applied multi-objective optimization procedure, used for determination of optimal tire design parameters for simultaneous minimization of strain energy density at belt edge and chafer is illustrated in Fig. 3. The procedure is divided into four main stages: pre-analysis, design of experiment (DOE), mathematical modeling and multi-objective optimization.

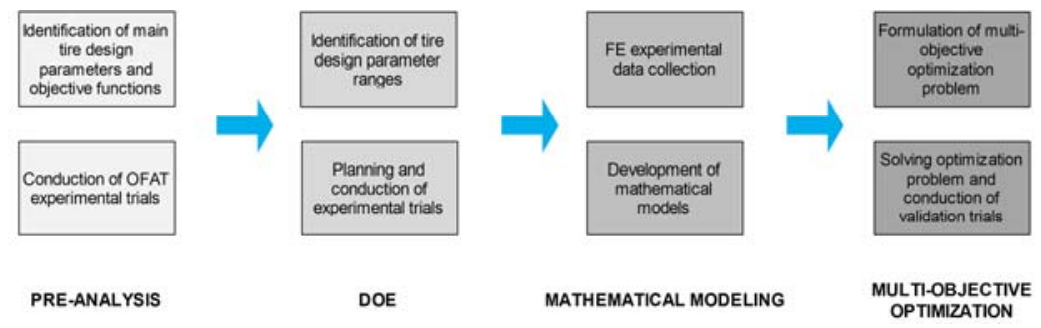

Fig. 3. Applied multi-response optimization procedure 
The goal of pre-analysis stage is to identify the main tire design parameters, which have a significant influence on the objective functions. As mentioned in previous chapter, for the purpose of pilot study, this phase was simplified by choosing three design parameters already reported to have significant influence on tire stresses. In order to investigate the sensitivity of objective functions to the change of selected tire design parameters values, one factor at a time (OFAT) experimental trials were performed in this stage.

In the DOE stage, considering manufacturing practices, data from literature (Olatunbosun \& Bolarinwa, 2004; Serafinska et al., 2013; Yang et al. 2014) and trial FE analyses, the ranges of values for tire design parameters were determined. In order to cover investigated experimental hyper-space, the full factorial experimental plan was used. Subsequently, based on different combinations of tire design parameter values, FE simulations were performed. In the mathematical modeling stage, based on the collected FE experimental data and by using the MRA, mathematical models relating tire design parameters and strain energy density at belt edge and chafer were developed and validated. The first step in multi-objective optimization stage was the formulation of the optimization problem, which was set as identification of optimal values of tire design parameters in order to simultaneously minimize strain energy density at belt edge and chafer. Subsequently, to obtain a set of Pareto optimal solutions, genetic algorithm (GA) was applied. Finally, for the purpose of validation, FE simulation experimental trials were performed with the identified optimal values of tire design parameters.

\section{Experimental plan and FE analyses results}

The accuracy of scientific experimentation can be increased by using the experimental plans from the DOE, which offer an efficient plan to study the entire experimental region of interest for the experimenter. Among the various DOE such as OFAT, factorial, fractional factorial, central composite design, Box-Behnken, Taguchi, etc., in this study a $3^{3}$ full factorial experimental plan was adopted. Although more time consuming than some other plans, this high resolution experimental plan was applied since it allows independent estimation of all main and interaction effects of design parameters, analysis of the interaction effects of design parameters and development of mathematical models of higher order. In the present experimental study, three tire design parameters, namely belt angle $\left(x_{d l}\right)$, belt cord spacing $\left(x_{d 2}\right)$, and elasticity of tread compound $\left(x_{d 3}\right)$, were considered. The tire design parameter ranges were selected based on preliminary OFAT results as well as by considering some technically manageable ranges and guidelines from literature. Here it should be noted that all tire design parameters can be considered as continuous design parameters i.e. can take any value within the specified ranges. For the experimentation purpose boundary points within the specified ranges of each tire design parameter were selected as low and high levels, while the centre level was taken at the middle of the range. The configuration of the initial design is defined as $x_{d 1}=22^{\circ}, x_{d 2}=1.05 \mathrm{~mm}$ and $x_{d 3}=1$. Tread compound was modeled using hyperelastic Yeoh material model. The value of $x_{d 3}=1$ corresponds to nominal values of Yeoh coefficients: $\mathrm{C}_{10}=1.0236 \mathrm{~N} / \mathrm{mm}^{2}, \mathrm{C}_{20}=-0.4272 \mathrm{~N} / \mathrm{mm}^{2}$ and $\mathrm{C}_{30}=0.1732 \mathrm{~N} / \mathrm{mm}^{2}$. Values of Yeoh coefficients used in various FE models were obtained by multiplication of all the coefficients with the value of $x_{d 3}$. Therefore, $x_{d 3}$ is dimensionless. Table 1 gives the ranges of tire design parameters and their levels within the experimentation.

\section{Table 1}

Tire design parameter ranges and their levels in the experiment

\begin{tabular}{|c|c|c|c|c|}
\hline \multirow{2}{*}{ Tire design parameter } & \multirow{2}{*}{ Unit } & \multicolumn{3}{|c|}{ Level } \\
\hline & & 1 & 2 & 3 \\
\hline Belt angle, $x_{d 1}$ & o & 18 & 22 & 26 \\
\hline Belt cord spacing, $x_{d 2}$ & $\mathrm{~mm}$ & 0.65 & 1.05 & 1.45 \\
\hline Elasticity of tread compound, $x_{d 3}$ & & 0.6 & 1 & 1.4 \\
\hline
\end{tabular}

Based on the selected tire design parameters and their levels, experimental design matrix was constructed in accordance with the standard $3^{3}$ full factorial experimental plan. The FE simulation results (Fig. 4) 
regarding objective functions $\left(f_{1}\right.$ and $\left.f_{2}\right)$, i.e. strain energy density at belt edge and chafer, based on each combination of tire design parameter values are given in Table 2.
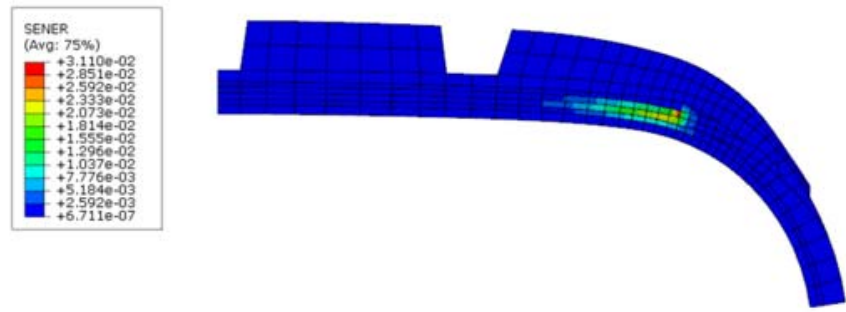

Fig. 4. Strain energy density obtained by FE analysis of axisymmetric model

Table 2

Experimental design and FE analysis simulation results

\begin{tabular}{|c|c|c|c|c|c|c|c|c|}
\hline \multirow[b]{2}{*}{$\begin{array}{l}\text { Exp. } \\
\text { trial }\end{array}$} & \multicolumn{3}{|c|}{$\begin{array}{c}\text { Combination of tire design } \\
\text { parameter levels }\end{array}$} & \multicolumn{3}{|c|}{ Real values of tire design parameter } & \multicolumn{2}{|c|}{$\begin{array}{l}\text { Objective } \\
\text { functions }\end{array}$} \\
\hline & $x_{d 1}$ & $x_{d 2}$ & $x_{d 3}$ & $\begin{array}{l}\text { Belt } \\
\text { angle } \\
\left({ }^{\circ}\right)\end{array}$ & $\begin{array}{l}\text { Belt cord } \\
\text { spacing } \\
(\mathrm{mm})\end{array}$ & $\begin{array}{c}\text { Elasticity of tread compound } \\
\text { (Yeoh coefficients } \\
\text { multiplication factor) }\end{array}$ & $\begin{array}{c}f_{l} \\
\left(\mathrm{~N} / \mathrm{mm}^{2}\right)\end{array}$ & $\begin{array}{c}f_{2} \\
\left(\mathrm{~N} / \mathrm{mm}^{2}\right)\end{array}$ \\
\hline 1 & 1 & 1 & 1 & 18 & 0.65 & 0.6 & 0.04640 & 0.03674 \\
\hline 2 & 1 & 1 & 2 & 18 & 0.65 & 1 & 0.03225 & 0.03666 \\
\hline 3 & 1 & 1 & 3 & 18 & 0.65 & 1.4 & 0.02997 & 0.03662 \\
\hline 4 & 1 & 2 & 1 & 18 & 1.05 & 0.6 & 0.04618 & 0.03684 \\
\hline 5 & 1 & 2 & 2 & 18 & 1.05 & 1 & 0.03227 & 0.03677 \\
\hline 6 & 1 & 2 & 3 & 18 & 1.05 & 1.4 & 0.03020 & 0.03673 \\
\hline 7 & 1 & 3 & 1 & 18 & 1.45 & 0.6 & 0.04595 & 0.03694 \\
\hline 8 & 1 & 3 & 2 & 18 & 1.45 & 1 & 0.03227 & 0.03688 \\
\hline 9 & 1 & 3 & 3 & 18 & 1.45 & 1.4 & 0.03038 & 0.03684 \\
\hline 10 & 2 & 1 & 1 & 22 & 0.65 & 0.6 & 0.07448 & 0.03872 \\
\hline 11 & 2 & 1 & 2 & 22 & 0.65 & 1 & 0.05341 & 0.03854 \\
\hline 12 & 2 & 1 & 3 & 22 & 0.65 & 1.4 & 0.05161 & 0.03841 \\
\hline 13 & 2 & 2 & 1 & 22 & 1.05 & 0.6 & 0.07303 & 0.03871 \\
\hline 14 & 2 & 2 & 2 & 22 & 1.05 & 1 & 0.05242 & 0.03855 \\
\hline 15 & 2 & 2 & 3 & 22 & 1.05 & 1.4 & 0.05076 & 0.03841 \\
\hline 16 & 2 & 3 & 1 & 22 & 1.45 & 0.6 & 0.07163 & 0.03870 \\
\hline 17 & 2 & 3 & 2 & 22 & 1.45 & 1 & 0.05147 & 0.03855 \\
\hline 18 & 2 & 3 & 3 & 22 & 1.45 & 1.4 & 0.04997 & 0.03843 \\
\hline 19 & 3 & 1 & 1 & 26 & 0.65 & 0.6 & 0.10730 & 0.04238 \\
\hline 20 & 3 & 1 & 2 & 26 & 0.65 & 1 & 0.08192 & 0.04213 \\
\hline 21 & 3 & 1 & 3 & 26 & 0.65 & 1.4 & 0.07753 & 0.04192 \\
\hline 22 & 3 & 2 & 1 & 26 & 1.05 & 0.6 & 0.10540 & 0.04234 \\
\hline 23 & 3 & 2 & 2 & 26 & 1.05 & 1 & 0.08039 & 0.04210 \\
\hline 24 & 3 & 2 & 3 & 26 & 1.05 & 1.4 & 0.07609 & 0.04189 \\
\hline 25 & 3 & 3 & 1 & 26 & 1.45 & 0.6 & 0.10350 & 0.04230 \\
\hline 26 & 3 & 3 & 2 & 26 & 1.45 & 1 & 0.07887 & 0.04207 \\
\hline 27 & 3 & 3 & 3 & 26 & 1.45 & 1.4 & 0.07470 & 0.04186 \\
\hline
\end{tabular}

All FE experimental data given in Table 2 were used for the development of objective functions in analytical form, regarding tire design parameters.

\section{Mathematical models for response surface approximation}

Mathematical models representing functional relationships between tire design parameters and performance characteristics allow for systematical analysis and optimization of tire design. ANN models were found to be very promising for empirical modeling of complex non-linearities and interactions in tire design (Nakajima et al., 1999; Koishi \& Shida, 2006; Serafinska et al., 2013). However, its practical application does not come without some shortfalls such as complex modeling procedure (numerous decisions related to ANN architectural and training parameters had to be made), lack of systematic design guidelines, high computational effort and time consuming approach. In cases where there are no such 
complex and highly nonlinear functional dependencies, it is more beneficial, in terms of speed and simplicity, to use simpler modeling methods such as MRA. Therefore, response surface approximation based on MRA was attempted. MRA is a conceptually simple method that when applied in tire design can be expressed by the following equation:

$$
f_{m}=f\left(x_{d 1}, \ldots, x_{d n} ; \beta_{0}, \ldots, \beta_{p}\right)+\varepsilon
$$

where $f_{m}$ is the objective function being modeled, $x_{d l}, \ldots, x_{n d}$ are tire design parameters, $\beta, \ldots, \beta_{p}$ are regression coefficients and $\varepsilon$ is the error.

\subsection{Development of MRA models}

To establish mathematical relationships between tire design parameters that is belt angle $\left(x_{d l}\right)$, belt cord spacing $\left(x_{d 2}\right)$ and elasticity of tread compound $\left(x_{d 3}\right)$, and strain energy density at belt edge and chafer, second order MRA models (quadratic regression models with interactions) were developed. By using the obtained FE experimental data and by the application of least square method for regression coefficients determination, the objective functions were obtained as:

$$
\begin{aligned}
f_{1}= & 0.030111+0.000741 \cdot x_{d 1}+0.008828 \cdot x_{d 2}-0.089748 \cdot x_{d 3}-0.000503 \cdot x_{d 1} \cdot x_{d 2} \\
& -0.002078 \cdot x_{d 1} \cdot x_{d 3}+0.000188 \cdot x_{d 1}^{2}+0.05366 \cdot x_{d 3}^{2} \\
f_{2}= & 0.0488-0.00167 \cdot x_{d 1} 0.00103 \cdot x_{d 2}+0.000813 \cdot x_{d 3}-0.000044 \cdot x_{d 1} \cdot x_{d 2} \\
& -0.000053 \cdot x_{d 1} \cdot x_{d 3}+0.000055 \cdot x_{d 1}^{2}
\end{aligned}
$$

More detailed results of MRA with all the corresponding coefficients and P-values are given in Tables 3 and 4. The adequacy of the developed MRA models was checked based on standard and adjusted coefficients of multiple determinations, $\mathrm{R}^{2}$ and $\mathrm{R}^{2}$ (adj.). The $\mathrm{R}^{2}$ values indicate that the tire design parameters explain more than $99 \%$ of variance in strain energy density. These values indicate that the developed models fit FE experimental data very well.

\section{Table 3}

The MRA model for the prediction of strain energy density at belt edge

\begin{tabular}{lcccc}
\hline Predictor & Coefficient & SE coefficient & $\mathrm{T}$ & $\mathrm{P}$ \\
\hline constant & 0.030111 & 0.015163 & 1.986 & 0.062 \\
$\mathrm{X}_{\mathrm{d} 1}$ & 0.000741 & 0.001281 & 0.579 & 0.570 \\
$\mathrm{X}_{\mathrm{d} 2}$ & 0.008828 & 0.004454 & 1.982 & 0.062 \\
$\mathrm{X}_{\mathrm{d} 3}$ & -0.089748 & 0.007205 & -12.456 & 0.000 \\
$x_{d 1}^{2}$ & 0.000188 & 0.000028 & 6.622 & 0.000 \\
$x_{d 3}^{2}$ & 0.053660 & 0.002832 & 18.948 & 0.000 \\
$\mathrm{X}_{\mathrm{d} 1} \cdot \mathrm{X}_{\mathrm{d} 2}$ & -0.000503 & 0.0002 & -2.512 & 0.021 \\
$\mathrm{X}_{\mathrm{d} 1} \cdot \mathrm{X}_{\mathrm{d} 3}$ & -0.002078 & 0.0002 & -10.378 & 0.000 \\
\hline $\mathrm{S}=0.001099 ; \mathrm{R}^{2}=0.9984$ & $\mathrm{R} 2$ & \\
\hline
\end{tabular}

$\mathrm{S}=0.0011099 ; \mathrm{R}^{2}=0.9984 ; \mathrm{R}^{2}(\mathrm{adj})=0.9978$. $\mathrm{T}$, value of Student's distribution; $\mathrm{P}$, probability density.

\section{Table 4}

The MRA model for the prediction of strain energy density at chafer

\begin{tabular}{lcccc}
\hline Predictor & Coefficient & SE coefficient & $\mathrm{T}$ & $\mathrm{P}$ \\
\hline constant & 0.048849 & 0.000258 & 189.608 & 0.000 \\
$\mathrm{X}_{\mathrm{d} 1}$ & -0.001675 & 0.000022 & -75.849 & 0.000 \\
$\mathrm{X}_{\mathrm{d} 2}$ & 0.001025 & 0.000077 & 13.355 & 0.000 \\
$\mathrm{X}_{\mathrm{d} 3}$ & 0.000813 & 0.000077 & 10.595 & 0.000 \\
$x_{d 1}^{2}$ & 0.000055 & 0.000000 & 113.623 & 0.000 \\
$\mathrm{X}_{\mathrm{d} 1} \cdot \mathrm{X}_{\mathrm{d} 2}$ & -0.000044 & 0.000003 & -12.678 & 0.000 \\
$\mathrm{X}_{\mathrm{d} 1} \cdot \mathrm{X}_{\mathrm{d} 3}$ & -0.000053 & 0.000003 & -15.395 & 0.000 \\
\hline $\mathrm{S}=0.0000191268 ; \mathrm{R}^{2}-0.999 ;$ & & & \\
\hline
\end{tabular}

$\mathrm{S}=0.0000191268 ; \mathrm{R}^{2}=0.999 ; \mathrm{R}^{2}(\operatorname{adj})=0.999$. 
In addition, the adequacy of FE experimental data fit by the developed MRA mathematical models was assessed using the absolute percentage errors. The mean absolute percentage errors between MRA model predictions and FE experimental data, for strain energy density at belt edge and chafer, were found to be $1.76 \%$ and $0.43 \%$ respectively. The obtained values as well as the results from Tables 3 and 4 suggest that the predictions of both MRA models are in very good agreement with FE experimental values of strain energy density within the scope of tire design parameter ranges investigated in the study. Thus, the developed MRA models can be used to analyze the effect of the tire design parameters on the strain energy density at belt edge and chafer. Also, MRA mathematical models can serve as objective functions for the optimization of tire design parameters.

\subsection{Effects of tire design parameters on strain energy density}

Initially effects of the tire design parameters on the strain energy density at belt edge and chafer were analyzed by changing one parameter at a time, while keeping the all other parameters constant at central level (level 2) (Fig. 5).

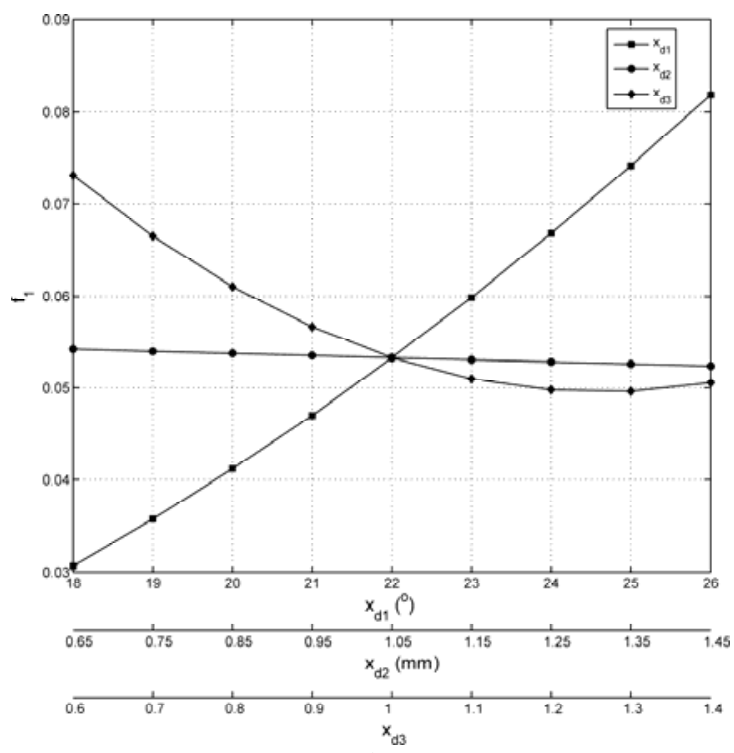

a)

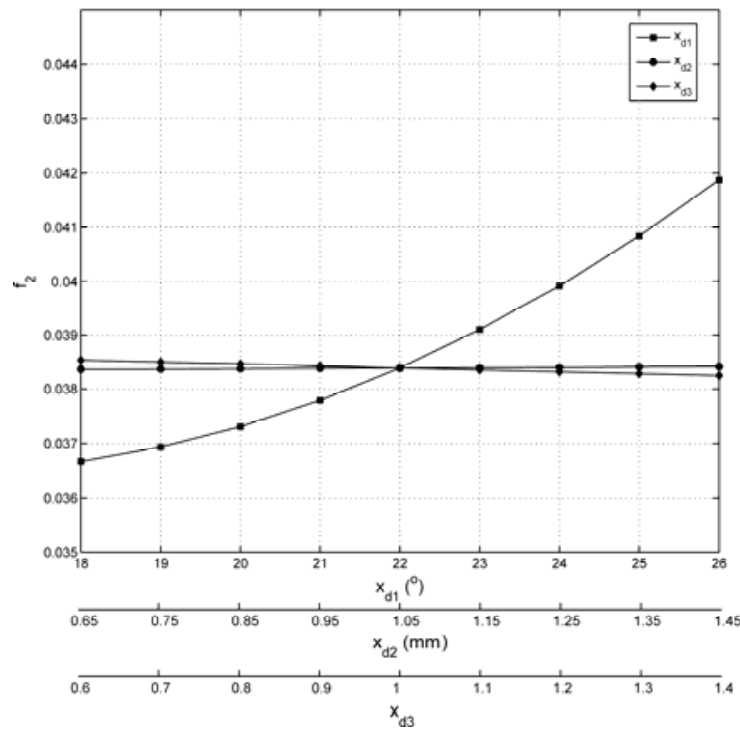

b)

Fig. 5. Main effects of the tire design parameters on the strain energy density at: a) belt edge, b) chafer

From Fig. 5 it can be seen that the increase in belt angle results in an increase in strain energy density. This is probably due to the fact that with increasing belt angle the angle between carcass and belt cord spacing becomes smaller and thus the stiffness change at belt edges becomes larger. On the other hand, for the range of tire design parameters investigated in the study, the influence of belt cord spacing on the strain energy density is negligible, although a small increase in strain energy density with decrease of belt cord spacing exists. The reason is probably also the increased stiffness change at belt edges. Further, it can be seen that strain energy density decreases with increasing elasticity of tread compound. This positive influence on minimization of the strain energy density can probably be attributed to less abrupt stiffness change in the vicinity of belt edge. From Fig. 5 it is clear that, quantitatively, the belt angle has the maximum influence on the strain energy density. Finally, it could be observed that the changes in the strain energy density at belt edge are significantly higher than in the chafer. This is due to the fact that chafer is situated much further from the zone of influence of selected tire design parameters. In order to determine the interaction effects of the tire design parameters on the strain energy density at belt edge and chafer, 3-D surface plots were generated considering two parameters at a time, while the third parameter was kept constant at center level. Since there are three possible two-way interactions ( $x_{d l}$ and 
$x_{d 2}, x_{d 1}$ and $x_{d 3}$, and $x_{d 2}$ and $x_{d 3}$ ), three 3-D plots were generated for strain energy density at belt edge (Fig. 6) and three 3-D plots were generated for the strain energy density at chafer (Fig. 6).

From Fig. 6 it can be seen that the increase in belt angle and decrease in belt cord spacing as well as increase in belt angle and decrease in elasticity of tread compound results in increase of the strain energy density at belt edge and at chafer. This is the expected combination of the separate influence of the two parameters. Fig. 6 confirms the negligible influence of the belt cord spacing in interaction with other tire design parameters on the strain energy density. From Fig. $6 \mathrm{c}$ and Fig. $6 \mathrm{f}$ it could be observed that decrease in elasticity of tread compound produces a nonlinear increase in strain energy density at belt edge, whereas the dependence of the strain energy density at chafer and elasticity of tread compound is linear.

\section{Pareto based optimization and results}

\subsection{Multi-objective optimization problem formulation}

The optimal selection of tire design parameters should increase tire durability to some extent by minimizing strain energy density at belt edge and chaffer. Therefore, one needs to define the multiobjective optimization problem, which in this study was formulated as follows:

$$
\begin{aligned}
& \text { Minimize: } f_{1}=f\left(x_{d 1}, x_{d 2}, x_{d 3}\right) \text { and } f_{2}=f\left(x_{d 1}, x_{d 2}, x_{d 3}\right), \\
& \text { subject to: } 18 \leq x_{d 1} \leq 26\left(^{\circ}\right), 0.65 \leq x_{d 2} \leq 1.45(\mathrm{~mm}), 0.6 \leq x_{d 3} \leq 1.4
\end{aligned}
$$

In the present multi-objective optimization procedure of tire design, two objective functions defined by Eq. (2a) and Eq. (2b) are considered and both are to be minimized.

\subsection{Solving approach}

Solving multi-objective optimization problems as the one formulated in Eq. (3) is quite difficult, because there is no unique solution; rather there exists a set of acceptable solutions. Methods for solving multiobjective optimization problems are usually divided into three categories: a priori methods, a posteriori methods and interactive methods, which involve active participation of a decision maker during the solving of an optimization problem and in essence combine a priori and a posteriori approaches (Deb, 2001).

In this study, a posteriori approach based on Pareto optimality concept was applied. Thus, determined optimal solutions are solutions, which are not dominated by any other solutions. The set of all Pareto optimal solutions is called the Pareto optimal set and the corresponding objective function vectors are said to be on the Pareto front (Ngatchou et al., 2005). As it is difficult to find Pareto solutions of multiobjective design problems of tires (Koishi \& Shida, 2006), genetic algorithm (GA), as one of the most powerful meta-heuristic optimization algorithms, was applied. GAs are powerful and broadly applicable probabilistic algorithms which combine elements of direct and stochastic search showing a high level of robustness (Michalewicz, 1996). The idea of GA is based on the principles of natural genetics and natural selection (Rao 2009). GAs have the advantage of evaluating multiple potential solutions in a single iteration. Moreover, they offer additional advantages such as greater flexibility for the decision maker, mainly in cases where no a priori information is available and handling non differentiable and discontinuous objective functions (Ngatchou et al., 2005), as well the ability to find solutions in a complex solution space quickly (Kovačević et al., 2014). For the purpose of optimization, the developed objective functions for the prediction of strain energy density were defined in MATLAB in m-files. Because of the stochastic nature of the GA, the optimization results are sensitive to main algorithm parameters. Hence, the next step was to select the main parameters of GA such as selection, crossover, 
mutation, population size, population type, and number of generations. In this study, repeated simulations were performed to find commensurate values for the main GA parameters (See Table 5).

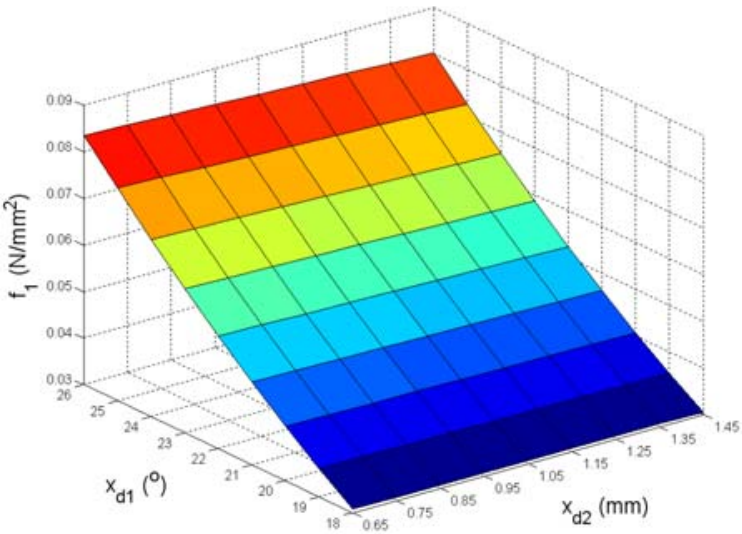

a)

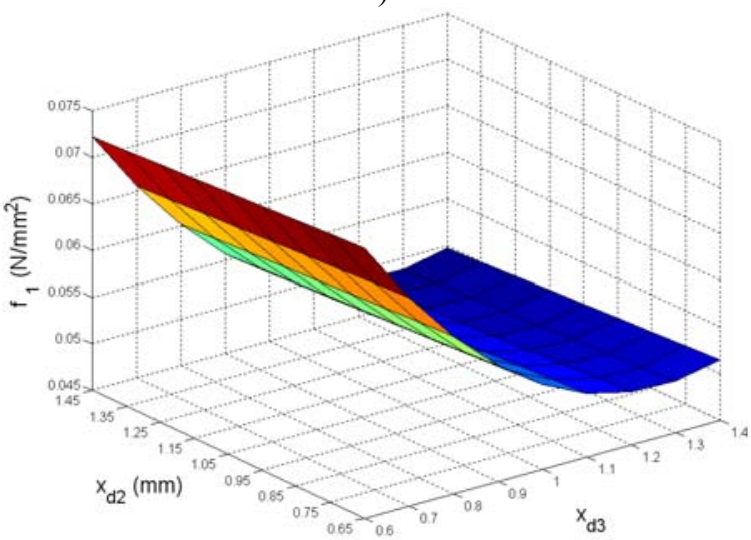

c)

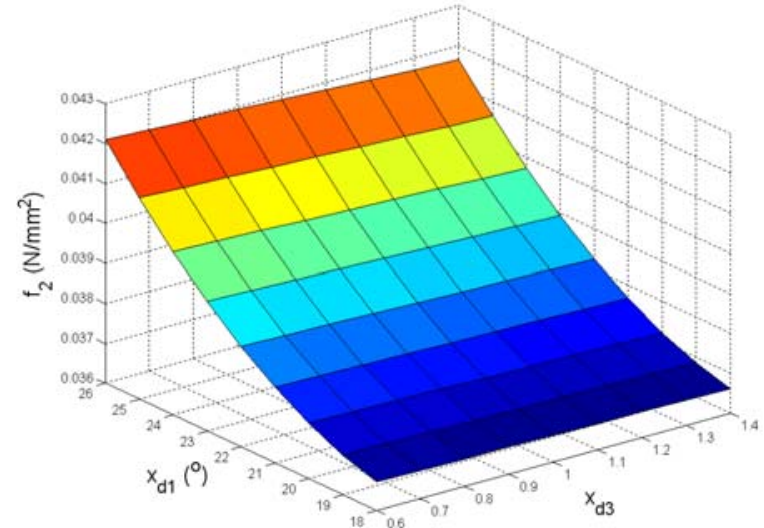

e)

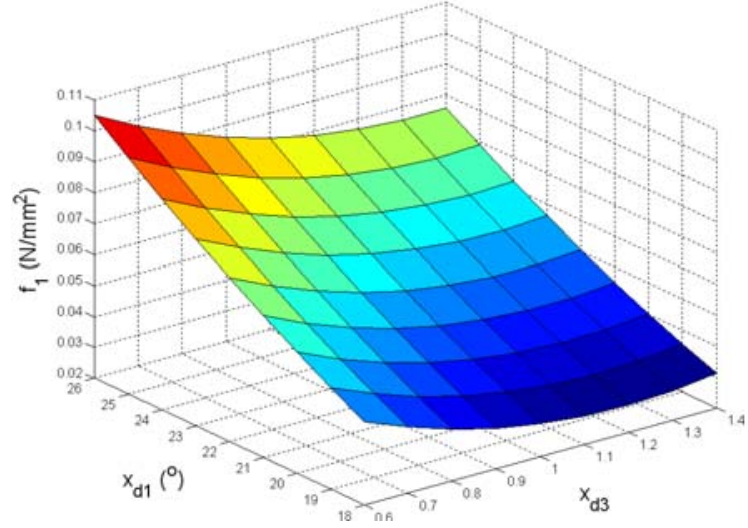

b)

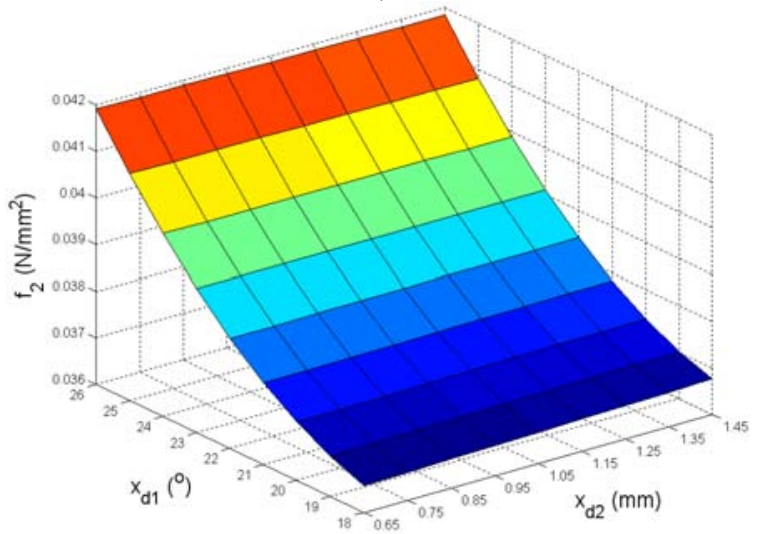

d)

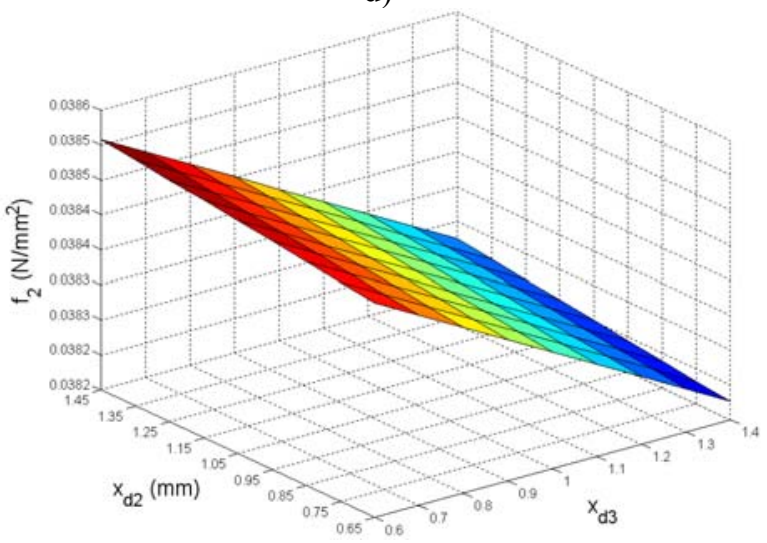

f)

Fig. 6. Interaction effects of the tire design parameters on the strain energy density at belt edge $(a, b, c)$

Table 5 and strain energy density at chafer $(\mathrm{d}, \mathrm{e}, \mathrm{f})$

Main parameter values of the GA used in the optimization process

\begin{tabular}{ll}
\hline Population size & 60 \\
Population type & double vector \\
Selection & stochastic uniform \\
Reproduction & elite count: 2 crossover fraction 0.9 \\
Crossover function & scattered \\
Mutation function & Gaussian \\
Max. generations & 800 \\
\hline
\end{tabular}

6.3. Multi-objective optimization solutions 
As a result of multi-objective optimization, a set consisting of 7 multi-objective optimal solutions was obtained based on the best fitness values (Fig. 7).

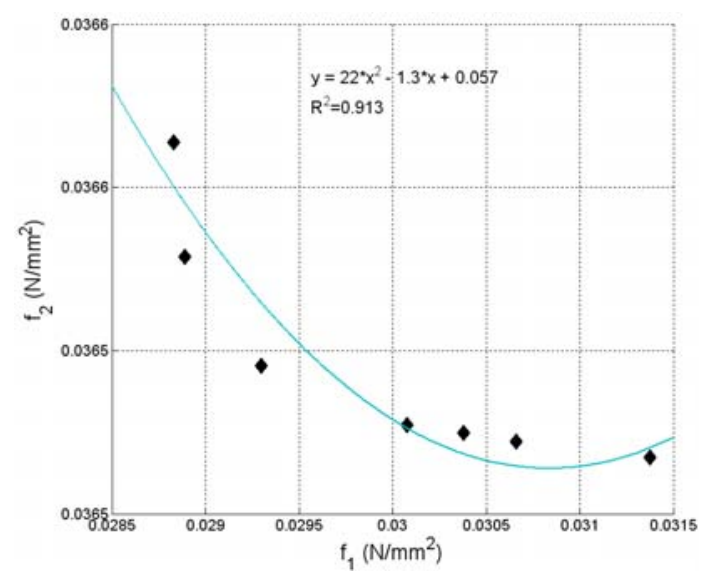

Fig. 7. The Pareto front of non-dominated solutions

As shown in Fig. 7, minimization of strain energy density at belt edge and minimization of strain energy density at chafer are contradicting objectives. However, it is obvious that the change in strain energy density at chafer is very small. From Fig. 7 it can be also observed that the relationship between strain energy density at belt edge and strain energy density at chafer is nonlinear and can be expressed with a second degree polynomial. None of the solutions in the Pareto-optimal front is absolutely better than any other, so as any one of them is an acceptable solution. The choice depends upon the specific design requirements and a suitable combination of tire design parameter values can be selected from Table 6 .

Table 6

Multi-objective optimal values of tire design parameters and corresponding values of strain energy density

\begin{tabular}{cccccc}
\hline Solution & $x_{d 1}\left({ }^{\circ}\right)$ & $x_{d 2}(\mathrm{~mm})$ & $x_{d 3}$ & $f_{1}\left(\mathrm{~N} / \mathrm{mm}^{2}\right)$ & $f_{2}\left(\mathrm{~N} / \mathrm{mm}^{2}\right)$ \\
\hline 1 & 18 & 0.65 & 1.4 & 0.031375 & 0.036517 \\
2 & 18 & 0.927 & 1.183 & 0.028828 & 0.036614 \\
3 & 18 & 0.653 & 1.351 & 0.030377 & 0.036525 \\
4 & 18 & 0.652 & 1.333 & 0.030076 & 0.036527 \\
5 & 18 & 0.692 & 1.273 & 0.029296 & 0.036545 \\
6 & 18 & 0.65 & 1.366 & 0.030659 & 0.036522 \\
7 & 18 & 0.795 & 1.209 & 0.028889 & 0.036579 \\
\hline
\end{tabular}

The analysis of the results from the Table 6 indicates that, for belt angle of $x_{d l}=18^{\circ}$, there are different combinations of belt cord spacing and elasticity of tread compound that yield acceptable solutions regarding simultaneous minimization of strain energy density at belt edge and at chafer. Note that the solution 1 actually corresponds to the experimental trial 3 from Table 2 . In order to check the quality of the obtained multi-objective optimization solutions, two independent single objective optimizations (minimizations) were performed. From the minimizations of the objective functions, the following results were obtained:

$$
\begin{aligned}
& f_{1 \min }=0.0287 \text { for } x_{d 1}=18^{\circ}, x_{d 2}=1.45 \mathrm{~mm}, x_{d 3}=1.185 \\
& f_{2 \min }=0.036517 \text { for } x_{d 1}=18^{\circ}, x_{d 2}=0.65 \mathrm{~mm}, x_{d 3}=1.4
\end{aligned}
$$

As it could be expected, single objective optimization results are somewhat better, in terms of objective function values, than the result of the multi-objective optimization. The trade-off between the optimization of two objective functions and the quality of the obtained solutions, when compared to the single objective optimization, is acceptable. Validation testing is necessary and final step in the applied 
multi-objective optimization procedure. Once the optimal combinations of tire design parameters are determined, the final step is to predict and verify the expected responses by performing the FE simulation experimental trials. Seven FE simulation experimental trials, with the combination of tire design parameter values as given in Table 6, were performed and the solution which had the minimal percentage error was selected as the best. It should be noted that the maximal percentage errors between FE simulation experimental values and predictions were less than 7\%. From Table 7 it can be observed that the there is a perfect match, with percentage errors less than $1 \%$, between predicted and FE experimental values of strain energy density. Consequently, this validation experimental trial gave excellent results and also revealed that the proposed multi-objective optimization approach had significantly improved the initial design (Table 8).

Table 7

Result of validation FE experimental trial

\begin{tabular}{lcc}
\hline & $f_{1}\left(\mathrm{~N} / \mathrm{mm}^{2}\right)$ & $f_{2}\left(\mathrm{~N} / \mathrm{mm}^{2}\right)$ \\
\hline FE simulation experiment & 0.03025 & 0.03663 \\
Predicted & 0.030377 & 0.036525 \\
percentage error, $\%$ & 0.4 & 0.3 \\
\hline Optimal combination of tire design parameter values: $x_{11}=18^{\circ}, x_{12}=0.653 \mathrm{~mm}, x_{13}=1.351$ &
\end{tabular}

Optimal combination of tire design parameter values: $x_{d 1}=18^{\circ}, x_{d 2}=0.653 \mathrm{~mm}, x_{d 3}=1.351$

\section{Table 8}

Comparison between the initial and optimal tire design parameter settings

\begin{tabular}{lccc}
\hline \multirow{1}{*}{ Tire design } & Tire design parameter values & \multicolumn{2}{c}{ Objective function values } \\
& & $f_{1}$ & $f_{2}$ \\
\cline { 2 - 4 } & & $x_{d 1}=22^{\circ}, x_{d 2}=1.05 \mathrm{~mm}, x_{d 3}=1$ & 0.05242 \\
\hline Initial design & $x_{d l^{2}}=18^{\circ}, x_{d 2}=0.653 \mathrm{~mm}, x_{d 3}=1.351$ & 0.03025 & 0.03855 \\
Optimized design & & 42.3 & 5 \\
Improvement $(\%)$ & & & 5 \\
\hline
\end{tabular}

\section{Conclusion}

In this paper, a procedure that integrates DOE, mathematical modeling and GAs for solving multiobjective optimization of tire design problems was proposed. In relation to the previous studies, the response surface approximation, used to evaluate objective functions in the optimization process, was obtained by the application of MRA. Apart from the fact that the use of response surface approximation can reduce the number of FE analyses i.e. CPU time dramatically, mathematical modeling of the relationships between tire design parameters and performance characteristics based on the use of MRA is much simpler and time saving approach. Furthermore, the proposed multi-objective optimization procedure is well suited for design engineers since it is based on the use of Pareto concept, thus providing a set of acceptable trade-of solutions of tire performance. The capabilities of the proposed procedure are illustrated through a design study. It is focused on determination of optimal tire design parameters for simultaneous minimization of strain energy density at belt edge and chafer. The results of the study are summarized in the following points:

- From the analysis of the effect of tire design parameters on the strain energy density at belt edge and chafer, it is observed that belt angle is the most significant tire design parameter followed by the elasticity of tread compound, while the influence of the belt cord spacing is negligible.

- The results of multi-objective optimization revealed that using small belt angle $\left(18^{\circ}\right)$ at suitable combination of belt cord spacing and elasticity of tread compound is favorable for minimization of strain energy density both at belt edge and at chafer.

- It was also observed that the strain energy density at chafer decreases nonlinearly as the strain energy density at belt edge increases and the functional dependence can be expressed with a second degree polynomial. 
- The proposed multi-objective optimization procedure resulted in significantly improved initial design. Improvements of $40 \%$ and $5 \%$ in objective function values were obtained and these were experimentally validated with percentage errors less than $1 \%$ between predicted and FE experimental values of strain energy density.

The practical application of the proposed multi-objective optimization procedure comes with increased flexibility on selecting the optimal tire design parameters by providing a set of candidate solutions for simultaneous consideration of several tire performance characteristics. Application of fractional factorial experimental plans for the analysis of several tire design parameters, including uncertain parameters, as well as the use of deterministic optimization methods for generation of Pareto solutions are future research scopes.

\section{References}

Cho, J. R., Jeong, H. S., \& Yoo, W. S. (2002). Multi-objective optimization of tire carcass contours using a systematic aspiration-level adjustment procedure. Computational Mechanics, 29(6), 498-509.

Deb, K. (2001). Multi-objective optimization using evolutionary algorithms. John Wiley \& Sons.

De Eskinazi, J., Ishihara, K., Volk, H., \& Warholic, T. C. (1990). Towards predicting relative belt edge endurance with the finite element method. Tire Science and Technology, 18(4), 216-235.

Gent, A. N., \& Walter, J.D. (2006). The pneumatic tire. Washington D.C., National Higway Traffic Safety Administration, U.S. Department of Transportation.

Ghoreishy, M. H. R. (2006). Finite element analysis of steady rolling tyre with slip angle: Effect of belt angle. Plastics, Rubber and Composites, 35(2), 83-90.

Koishi, M., \& Shida, Z. (2006). Multi-objective design problem of tire wear and visualization of its Pareto solutions, Tire Science and Technology, 34(3), 170-194.

Korunović, N., Trajanović, M., \& Stojković, M. (2007). Finite element model for steady-state rolling tire analysis. Journal of the Serbian Society for Computational Mechanics, 1(1), 63-79.

Kovačević, M., Madić, M., Radovanović, M., \& Rančić, D. (2014). Software prototype for solving multiobjective machining optimization problems: Application in non-conventional machining processes. Expert Systems with Applications, 41(13), 5657-5668.

Michalewicz, Z. (1996). Genetic algorithms + data structures= evolution programs. Springer.

Nakajima, Y., Kadowaki, H., Kamegawa, T., \& Ueno, K. (1999). Application of a neural network for the optimization of tire design. Tire Science and Technology, 27(2), 62-83.

Ngatchou, P., Zarei, A., \& El-Sharkawi, M. A. (2005). Pareto multi objective optimization. In Intelligent Systems Application to Power Systems, 2005. Proceedings of the 13th International Conference on (pp. 84-91). IEEE.

Olatunbosun, O. A., \& Bolarinwa, O. (2004). FE simulation of the effect of tire design parameters on lateral forces and moments. Tire science and Technology, 32(3), 146-163.

Rao, S. S. (2009). Engineering optimization: theory and practice. John Wiley \& Sons.

Serafinska, A., Kaliske, M., Zopf, C., \& Graf, W. (2013). A multi-objective optimization approach with consideration of fuzzy variables applied to structural tire design. Computers and Structures, 116, 719.

Yang, X., Behroozi, M., \& Olatunbosun, O. A. (2014). A Neural Network Approach to Predicting Car Tyre Micro-Scale and Macro-Scale Behaviour. Journal of Intelligent Learning Systems and Applications, 6, 11-20. 